Nouvelles perspectives en sciences sociales

\title{
Sciences sociales et approche relationnelle
}

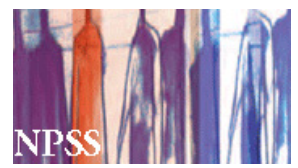

\section{Simon Laflamme}

Volume 5, numéro 1, octobre 2009

Sur le thème de la relation

URI : https://id.erudit.org/iderudit/038623ar

DOI : https://doi.org/10.7202/038623ar

Aller au sommaire du numéro

\section{Éditeur(s)}

Prise de parole

\section{ISSN}

1712-8307 (imprimé)

1918-7475 (numérique)

Découvrir la revue

\section{Citer cet article}

Laflamme, S. (2009). Sciences sociales et approche relationnelle. Nouvelles perspectives en sciences sociales, 5(1), 79-85. https://doi.org/10.7202/038623ar

\section{Résumé de l'article}

La sociologie, comme l'économie dont elle est issue, et comme la plupart des sciences sociales, oscille entre deux approches : l'une qui veut que les phénomènes sociaux soient interprétables en fonction d'un acteur rationnel, l'autre d'après laquelle l'explication des phénomènes sociaux résulte de la possibilité d'en saisir les relations. Ces deux perspectives cohabitent souvent, même au sein d'une même théorie; mais elles sont contradictoires : le social ne peut être le strict produit d'actions individuelles en même temps que celui des interactions humaines ou des relations entre les phénomènes sociaux. C'est pourquoi l'interactionnisme s'impose aux théories individualistes et pourquoi aussi l'idéologie d'un acteur rationnel, libre, intéressé, intentionnel et stratégique n’annule pas toutes les démarches relationnelles. Les approches relationnelles sont essentielles aux sciences sociales bien simplement parce qu'il n'y a pas d'individu en dehors d'interactions humaines et qu'il n'y a pas non plus de phénomène social qui ne soit pas ou bien un composé de relations ou bien lui-même en relation avec d'autres phénomènes. 


\title{
Sciences sociales et approche relationnelle
}

\author{
Simon LAFLAMme \\ Université Laurentienne, Sudbury
}

Tes sciences sociales naissent avec l'économie politique. —L'économie politique porte en elle deux perspectives : l'une qui veut que l'acteur économique agisse de façon rationnelle, c'est-à-dire en fonction de son intérêt; l'autre d'après laquelle les constituants de l'économie agissent les uns par rapport aux autres, la demande en fonction de l'offre, par exemple, ou encore la richesse par rapport à l'accumulation de capital, la division du travail et la taille du marché. D'un côté, donc, la science économique adopte une vision non relationnelle, celle d'un individu autonome qui agit en fonction de ses besoins de manière stratégique; de l'autre, elle s'inscrit dans une approche où les variations d'un phénomène s'expliquent en fonction des variations d'autres phénomènes.

La sociologie, à ses origines, fait largement la même chose. Chez Marx, elle propose un acteur économique intéressé, conscient - à moins qu'il ne soit aliéné -, en même temps que des composantes sociales qui se déterminent entre elles : l'économique, le politique et le religieux s'influençant mutuellement dans les écrits les plus lucides, l'économique déterminant unilatéralement tout autre aspect de la société dans les textes les plus 
naifs. Chez Weber, la sociologie se construit à nouveau sur un acteur libre, intéressé, intentionnel, vision qui se veut l'assise de la méthodologie qui y est développée; parallèlement, elle observe que les comportements religieux ont une incidence sur l'agir économique, et donc sur l'évolution du capitalisme, tout en admettant que l'économique puisse moduler les attitudes religieuses. Chez Durkheim, l'ambivalence est moins évidente : la sociologie se livre dans une analogie avec la biologie où les divers organes de la société fonctionnent les uns par rapport aux autres; mais elle décrit aussi, à la périphérie, des individus qui, sous l'effet des déterminants sociaux, agissent dans leur intérêt.

Dès leurs débuts, donc, quand des sciences sociales comme l'économie - qui sera au fondement de plusieurs autres disciplines - et la sociologie étudient la société sans se donner pour objet la personne humaine, elles n'hésitent pas à expliquer les modifications d'un objet en fonction de celles qui s'opèrent dans un autre objet. Il est donc compris que les objets sociaux existent et évoluent les uns par rapport aux autres, que leur autonomie ne peut être que relative. Le structuralisme, la systémique, l'analyse complexe ne feront que peaufiner cette position épistémologique aussi bien par leurs développements théoriques - dans lesquels, par exemple, on note que l'idée de réciprocité occupe de plus en plus d'espace par comparaison à celle de causalité linéaire - que par leurs observations, c'est-à-dire par la construction de l'empirie à laquelle elles se donnent accès. Intuitivement, cela se comprend aisément : nul objet social n'existe dans une pure indépendance à l'égard des autres; la division du travail, par exemple, dépend de la structure familiale, et inversement; elles deux sont en interaction avec la manière dont est géré le politique. Et ainsi de suite.

Dès leurs débuts, toutefois, les sciences sociales ont de la difficulté à appliquer à l'humain ce principe d'interdépendance des objets sociaux. L'humain, c'est d'ailleurs l'individu. Cet individu se présente à elles comme un être autonome, ainsi que l'a dépeint une longue tradition philosophique. Il est autonome parce que conscient, intentionnel et rationnel. La concomitance des termes 
servant souvent de preuve de la pertinence de chacun d'eux : il est juste de parler d'intention puisque l'humain est conscient; il est légitime de parler de conscience puisque l'humain est rationnel; et ainsi de suite dans une logique en circuit fermé. Les sciences sociales représentent donc communément une affirmation de l'autonomie de l'individu. Elles développent de beaux modèles, découvrent maintes vérités, sans avoir pour centre l'individu; et elles jurent de la liberté de l'individu. Maintes fois, d'ailleurs, elles ont pour motif de se rebeller contre les approches qui ne mettent pas l'individu au centre des analyses et qui, ce faisant, ne déclarent pas l'individu comme libre. Tels sont bien les milliers de travaux qui sont réalisés dans l'esprit de l'interactionnisme symbolique, de l'ethnométhodologie, de l'ethnographie institutionnelle et de l'individualisme méthodologique. Ces mêmes travaux ne sont pas exempts de contradictions : s'ils sont sans conteste des plaidoyers pour un acteur social libre, conscient, intentionnel, rationnel; au plan empirique, ils sont couramment la démonstration non assumée de ce que ce qui advient socialement est davantage le produit des relations humaines que celui de la volonté d'un acteur et de ce que ces relations humaines dépendent de circonstances sur lesquelles elles ont une incidence. L'interactionnisme symbolique est effectivement un interactionnisme, beaucoup plus qu'il ne constitue la preuve que tout acteur social passe son temps à volontairement sauver la face! L'ethnométhodologie est beaucoup moins la preuve que les acteurs sont toujours conscients et qu'ils ne subissent pas l'influence des structures sociales qu'elle est la démonstration par ses innombrables analyses que les acteurs agissent sur le mode de la circonstance les uns par rapport aux autres. L'économie, comme bon nombre de disciplines, porte en elle un acteur libre; mais elle le maintient à la périphérie dans bon nombre de ses travaux. La théorie de l'offre et de la demande part du principe que l'acteur économique agit d'après son intérêt; mais, en même temps, elle se développe mathématiquement sans référence au principe. Ce qui est étonnant par-dessus tout, c'est que les sciences sociales, malgré qu'elles se fassent couramment les 
chantres de l'individu et de sa liberté, découvrent avec une évidence croissante :

i. que tout individu est toujours en lien avec d'autres et que même l'ermitage est un type particulier de relation aux autres;

ii. que la psyché d'un individu est le produit dynamique des relations humaines;

iii. que la psyché d'un individu se construit sur le langage qui est forcément une désubjectivation;

iv. que la psyché d'un individu n'est pas toujours conscience;

v. que les actions des individus dépendent de circonstances sociales et historiques;

vi. que maints comportements individuels ne sont pas conscients, rationnels ou intentionnels;

vii. que tout entendement de la socialité ne passe pas par l'individu.

Bref, les sciences sociales constatent que tout acteur social est toujours en relation avec d'autres et avec le milieu symbolique au sein duquel il évolue. Elles découvrent donc que la notion d'autonomie est inopérante, que si elle peut constituer une catégorie idéologique, elle ne peut certainement pas jouer le rôle de concept au sein d'une théorie qui accepte de se soumettre à l'opérationnalisation. (S'il est vrai, au passage, qu'on peut associer liberté humaine à rationalité, il est exagéré d'y voir là une corrélation nécessaire; l'humanité n'est pas d'autant plus libre que les individus sont rationnels.) Les sciences sociales remarquent aussi qu'on peut étudier le social en suivant de près les actions humaines, mais qu'on peut tout aussi bien se pencher sur les phénomènes sociaux sans s'arrêter sur les individus, par exemple en se concentrant sur des institutions ou même sur des catégories plus abstraites comme l'échange ou la structure, ou la dynamique, ou la vitalité, ou la redondance... Cela ne constitue pas pour autant une négation du fait qu'il y ait des acteurs sociaux dans une société. 
Les sciences sociales ne sont pas d'autant plus scientifiques qu'elles réduisent l'acteur social au mode de l'intérêt; car elles omettent alors les innombrables manifestations de l'humanité qui se révèlent en dehors de la conscience et de l'intention, laissant échapper tout ce qui est de l'ordre de l'émotion par exemple. Les sciences sociales ne sont pas d'autant plus justifiables qu'elles affirment la liberté humaine; car la liberté est davantage une catégorie idéologique qu'une catégorie scientifique. Les sciences sociales ne sont pas d'autant plus autorisées qu'elles examinent les individus; car il ne s'agit là que d'une possibilité analytique parmi d'autres. Le problème des sciences sociales, ce n'est pas qu'elles mettent trop l'accent sur l'individu, c'est que, en le faisant, elles enfoncent leur objet dans des catégories phénoménologiques, c'est qu'une logique de circonstances empiriquement observables devient un axiome, une précompréhension de l'agir humain; si l'humain peut agir de façon intentionnelle, consciente et rationnelle, il peut aussi très bien ne pas le faire. Les sciences sociales doivent être en mesure d'appréhender tous ces agir. Les sciences sociales ont beaucoup plus de difficulté avec la théorisation qu'avec l'observation. La vision individualisante en est largement responsable. Les sciences sociales sont parfaitement équipées pour décrire le social; la grande majorité des spécialistes savent enquêter sur le social, de façon qualitative ou quantitative; et le sont mieux que quiconque ceux parmi eux qui savent manipuler aussi bien les outils qualitatifs que les autres. Si trop de spécialistes des sciences sociales sont de mauvais méthodologues, ce n'est pas en tant qu'ils ne savent pas utiliser leurs instruments de collecte de données préférés, c’est parce qu'ils ne savent pas articuler problématique, hypothèses et méthode; c'est parce qu'ils font un usage idéologique de leurs outils de collecte de données. C'est parce que la vision individualisante rend possible trop souvent une sociologie militante, parce qu'elle fournit des coupables intéressées et des victimes, parce qu'elle permet que trop de sociologues confondent leur discipline et les causes humanitaires auxquelles ils adhèrent. Si la sociologie 
a pour motif, et non pour objet, les individus, alors elle appelle les sauveurs. Si la sociologie a pour motif les individus, alors elle est amenée à éviter l'abstraction grâce à laquelle les sciences peuvent évoluer et, partant, se désubjectiver.

$\grave{A}$ un premier niveau, l'approche relationnelle, pour nous, est celle qui reconnaît que les objets sociaux agissent les uns par rapport aux autres et que l'autonomie de chacun d'eux ne peut être que relative. C'est aussi celle qui soutient que les individus doivent être compris dans les relations qu'ils entretiennent avec les autres et avec leur environnement social et historique. À un second niveau, l'approche relationnelle admet la possibilité de la causalité unilatérale, mais elle est ouverte aux phénomènes de réciprocité où peuvent interagir plusieurs objets plus ou moins abstraits dont tous n'appartiennent pas forcément à une logique monodisciplinaire. C'est aussi celle qui refuse que l'esprit humain soit réduit à une modélisation phénoménologique qui dissout toute la psyché dans un appareillage conceptuel fait d'intention, de conscience, d'autonomie et de raison; pour elle, l'humain est parfois intentionnel, parfois non, parfois conscient, parfois non, et, si l'on tient à la catégorie, parfois libre, parfois non; dans sa quotidienneté, il n'est pas purement rationnel parce que sa raison cohabite avec l'émotion, il est simultanément rationnel et émotif, et c'est pourquoi nous avons proposé la catégorie d' "émoraison ". À un troisième niveau, l'approche relationnelle que nous préconisons privilégie l'abstraction, car c'est à travers elle que les sciences sociales peuvent effectivement devenir des sciences et non pas des plaidoiries ou des outils militants, c'est à travers l'abstraction que les sciences peuvent construire des modèles qui acceptent réellement de se soumettre à l'évidence de l'empirie; c'est dans cet esprit que nous avons tenté de comprendre le Canada en dehors des logiques politiques ou territoriales en modélisant de façon trialectique la circulation des biens, des idées et des personnes. L'approche relationnelle à laquelle nous souscrivons met aussi l'accent sur le lien plutôt que sur les objets qui sont reliés; car tout objet n'existe qu'à travers sa relation aux autres : elle ne nie pas, dans le cadre des relations entre les per- 
sonnes, par exemple, que ces personnes puissent agir les unes sur les autres ou qu'on puisse les étudier séparément; mais elle met l'accent sur la dynamique puisqu'il n'y a pas de subjectivité qui ne soit pas informée et que la circulation de l'information procède de relations humaines; il n'y a pas d'humanité en dehors du champ symbolique, donc de l'information et de la communication, et la communication procède des relations humaines; l'interprétation à laquelle procède une subjectivité est elle-même un mouvement qui est indéfinissable en dehors du champ des dynamiques sociales; les valeurs, les normes viennent toutes à la personne à travers la communication, sous toute ses formes, donc à travers les relations humaines. L'action humaine est incompréhensible en dehors de l'interaction. L'acteur social est actif dans le champ des relations; mais son action procède de relations. 